\title{
Beta-Asarone Alleviates Myocardial Ischemia-Reperfusion Injury by Inhibiting Inflammatory Response and NLRP3 Inflammasome Mediated Pyroptosis
}

\author{
Bin Xiao, ${ }^{a}$ Xiaobo Huang, ${ }^{*}, a$ Qian Wang, ${ }^{a}$ and Yanchuan $\mathrm{Wu}^{b}$ \\ ${ }^{a}$ Department of Traditional Chinese Medicine, Xuanwu Hospital Capital Medical University; Beijing 100053, P. R. \\ China: and ${ }^{b}$ Central laboratory of Xuanwu Hospital Capital Medical University; Beijing 100053, P. R. China. \\ Received October 20, 2019; accepted March 27, 2020; advance publication released online April 22, 2020
}

Beta-asarone ( $\beta$-Asarone), the major component of Acorus tatarinowii Rhizoma, has been proved to be muti-pharmacological activities including anti-inflammation, and which is effective in protecting the central nervous system. However, the effect of $\beta$-Asarone on myocardial ischemia-reperfusion (I/R) injury is not yet clear. This study used a rat model with $45 \mathrm{~min}$ occlusion and $24 \mathrm{~h}$ releasing of proximal segment of left anterior descending coronary artery. The effects of $\beta$-Asarone on cardiac histopathology, myocardial infarc-

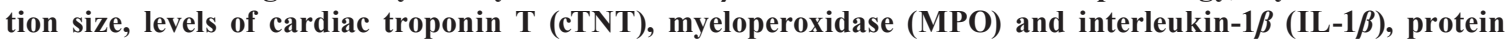
expressions of apoptosis-associated speck-like protein containing a CARD (ASC), Nod-like receptor protein 3 (NLRP3), caspase-1 and Gasdermin D (GSDMSD), and left ventricular performance were studied respectively. Our results showed that administration of $\beta$-Asarone significantly improved the heart outcome after myocardial ischemia and reperfusion in terms of less infarction size and lower serum cTNT concentration. Further, $\beta$-Asarone treatment evidently inhibited inflammatory response with less granulocyte infiltration, mild tissue edema and lower tissue MPO content, it also suppressed NLRP3 signal pathway and cardiac cell's pyroptosis for less protein expressions of ASC and NLRP3, lower level cleavage activation of caspase-1 and GSDMSD, and lower serum IL-1 $\beta$ concentration. Finally, $\beta$-Asarone treatment well preserved the left ventricular performance with higher ejection fraction and fractional shortening. The experimental results suggested that $\beta$-Asarone was protective against myocardial ischemia-reperfusion injury, in which inhibition of inflammatory response and suppression of NLRP3 inflammasome mediated pyroptosis were supposed to play a vital role.

Key words beta-asarone; myocardial reperfusion injury; inflammation; nod-like receptor protein 3 inflammasome; pyroptosis

\section{INTRODUCTION}

With the aging process of whole population due to development of economy and society, acute myocardial infarction (AMI) has become a widespread public health problem for its high mortality. ${ }^{1)}$ Although reperfusion therapy is widely accepted as the critical measure for AMI, studies have observed that reperfusion itself may bring additional myocardial damage, which is more serious than caused by ischemia alone, termed as "ischemia-reperfusion (I/R) injury."2) Myocardial $\mathrm{I} / \mathrm{R}$ injury has many facets, but cardiac cell necrosis or cell death, namely lethal reperfusion injury, is the core issue because it has more clinical significance, always meaning a worse outcome. ${ }^{3)}$ Even basic experiments on myocardial I/R injury discovered a lot of pathophysiological mechanisms and yielded encouraging results after taking intervention on them, the actual effects of those managements in clinical treatment were not desirable., ${ }^{4,5}$ The lack of effective and widely applicable treatments promoted a growing interest in traditional medicines. $\beta$-Asarone (cis-2,4,5-trimethoxy-1-allyl phenyl), is the major active component of a trditional Chinese medicine-Acorus tatarinowii Rhizoma. ${ }^{6}$ It had been repeatedly proved that $\beta$-Asarone was effective in protecting the central nervous system, in which its anti-inflammation activity played an important part. ${ }^{7,8)}$ As inflammatory response and dysimmunity were also important pathomechanisms of reperfusion induced cardiac damage, ${ }^{4}$ we hypothesize that $\beta$-Asarone possess protective effect against myocardial I/R injury.

\section{MATERIALS AND METHODS}

Animals Healthy male Sprague-Dawley rats with each body weight of $240-280 \mathrm{~g}$ were used in the study. All animals were purchased from Vital River Lab Animal Technology Co., Ltd. (Beijing, China), and kept under standard conditions of temperature $\left(22 \pm 2^{\circ} \mathrm{C}\right)$, relative humidity $(50 \pm 10 \%)$, and light $(12 / 12 \mathrm{~h} \mathrm{light/dark}$ cycle). Rats had free access to a standard rodent diet ad libitum. This study was approved by Xuanwu Hospital Capital Medical University's ethics committee, and all procedures complied with the guidance set out in the Guidelines for Caring for Experimental Animals published by the Ministry of Science and Technology of the People's Republic of China. Every care was taken to minimize discomfort, distress and pain.

Chemicals $\beta$-Asarone with the purity of over $98 \%$ were bought from PUSH BIO-TECONOLOGY (Chengdu, China).

Main Reagents and Kits Cardiac troponin $\mathrm{T}$ (cTNT), myeloperoxidase (MPO) and interleukin-1 $\beta$ (IL-1 $\beta$ ) enzymelinked immune sorbent assay (ELISA) kits were bought from Expandbiotech (Beijing, China). Primary antibodies for Western blot were obtained as follows: apoptosis-associated speck-like protein containing a CARD (ASC) and caspase-1 
from Wanleibio (Shenyang, China), gasdermin D (GSDMSD) from Cell Signaling Technology (Beverly, MA, U.S.A.), glyceraldehyde-3-phosphate dehydrogenase (GAPDH) from Immunoway (Plano, TX, U.S.A.), and Nod-like receptor protein 3 (NLRP3) from Abcam (Cambridge, U.K.). Anti-rabbit HRPconjugated immunoglobulin $\mathrm{G}$ ( $\mathrm{IgG}$ ) secondary antibody was purchased from Sigma-Aldrich (St. Louis, MO, U.S.A.). Evans blue and 2,3,5-triphenylte trazolium chloride (TTC) reagent were bought from Amresco (Missouri, MO, U.S.A.). All other chemicals and solvents were of analytical grade.

Experimental Protocol After three days of acclimation, the animals were divided randomly into five groups of six rats each. The first group served as sham (Sham). The second group was the ischemia-reperfusion (I/R) group. Group I and Group II were treated by normal saline (NS) respectively. Group III (low dose (LD)), Group IV (medium dose (MD)) and Group V (high dose (HD)) were I/R treated by $\beta$-Asarone with 10,20 , and $30 \mathrm{mg} / \mathrm{kg}$, respectively. The myocardial I/R model was established according to previous literatures. ${ }^{9)}$ Briefly, rat was anesthetized with $10 \%$ chloral hydrate of $3.5 \mathrm{~mL} / \mathrm{kg}$ by peritoneal injection, then thorax were opened through left 3-4th intercostal muscles. After exposure of the heart clearly, the animal was subjected to the occlusion of proximal segment of left anterior descending coronary artery (LAD) for $45 \mathrm{~min}$, followed by $24 \mathrm{~h}$ reperfusion with chest closed. The sham group was exposed to a time-matched normal perfusion followed by threading the LAD without occlusion. NS or $\beta$-Asarone was administrated by intraperitoneal injection just before loosening of the ligation. The operation was supported by mechanical ventilation and electrocardiogram monitoring. All animals were received jugular vein catheterization for collection of blood sample or drug administration.

Evaluation of Myocardial Infarction Size The myocardial infarction size was evaluated by TTC-Evans blue double staining as previously described. ${ }^{10)}$ Briefly, each rat was slowly injected with $1 \mathrm{~mL} 1 \%$ TTC solution from the jugular catheter. Thereafter the LAD was ligated in situ again, and $2 \mathrm{~mL} 1 \%$ Evans blue solution was injected by the same way. Then the heart was taken out when the animal's lip became blue. After the excess dyes washed and water sucked out, the specimen were sent to rapid freezing at $-20^{\circ} \mathrm{C}$ for $30 \mathrm{~min}$. Then each heart was cut into five pieces equably at the line which was parallel to the coronary sulcus and below the ligation knot. In the stained sections, the normal tissues were stained blue, and the ischemic risk regions were stained red, while the infarcted areas remained unstained (white or pale). The slices were photographed immediately and analyzed by Image-Pro Plus image analysis software (Version 4.1, Media Cybernetics, U.S.A.). Extents of myocardial infarction (necrosis area/area at risk $\%$, NA/AAR\%) were calculated and recorded.

Determination of cTNT, MPO and IL-1 $\beta$ Levels Serum cTNT, IL-1 $\beta$ and cardiac tissue MPO levels were quantified using commercial ELISA kits according to the manufacturer's instructions.

Histological Examination Immediately after sacrifice of the rats, the cardiac tissues were removed and fixed in $4 \%$ paraformaldehyde solution. The specimens were processed for sectioning and staining by standard histological methods. Sections from the left ventricle were stained with hematoxylineosin (Solarbio, China) reagent and examined by light microscopy at $200 \times$ magnification.
Western Blot Analysis Cardiac protein extraction was extracted using the cytoplasm protein extraction kit, and the protein contents of the cytoplasmic extracts were determined with the bicinchoninic acid (BCA) method (Wanleibio, China). Equal amounts of $40 \mu \mathrm{g} /$ lane of protein were subjected to sodium dodecyl sulfate polyacrylamide gel electrophoresis (SDS-PAGE) and transferred to polyvinylidene fluoride membranes. Blots were blocked for $1 \mathrm{~h}$ in $5 \%$ nonfat dry milk-tris buffered saline (TBS)-0.1\% Tween 20 and then washed. Primary antibodies were incubated overnight at $4^{\circ} \mathrm{C}$ followed by a horseradish peroxidase-conjugated secondary anti-rabbit antibody (1:5000) for $45 \mathrm{~min}$. Immunoreactivity was detected through the enhanced chemiluminescence detection reagents by a gel imaging system.

Examination of Left Ventricular Performance Rats were anesthetized and fixed on the checking table with exposure of the chest skin. Using Vevo 2100 ultrasonic cardiograph machine (VisualSonics, Canada) and a $30-\mathrm{MHz}$ small animal probe, the following parameters were recorded: left ventricular end systolic diameter (LVIDs), left ventricular end diastolic diameter (LVIDd), left ventricular ejection fraction (LVEF) and left ventricular fractional shortening (FS). Each of the parameter was measured 3 times continuously and recorded the average value as experimental data.

Statistical Analysis All values were normally distributed and expressed as the mean \pm standard deviation (S.D.). and were analyzed by a Student's test. In the case of multiple mean comparisons, the data were analyzed by one-way ANOVA followed by Duncan's Multiple Range Test using SPSS15.0 software. The differences were regarded significant when $p<0.05$, and statistically very significant if $p<0.01$. All statistical graphs were depicted by Graphpad8.0 software.

\section{RESULTS}

Results of Animal Myocardial I/R Model Rats were ligated at proximal segment of LAD reversibly by surgical operation. There were dynamic changes of electrocardiogram (ECG) and heart surface color along with the processing, suggesting an established myocardial ischemia followed by efficient restoration of blood flow (Fig. 1).

Effects of $\boldsymbol{\beta}$-Asarone on the Size of Myocardial Infarction Rats with myocardial I/R stress presented evident infarction lesions in TTC-Evans blue double stained specimens. Compared with the $\mathrm{I} / \mathrm{R}$ control group, $\beta$-Asarone treatment reduced myocardial infarction size as lower NA/AAR\%, especially in the MD and HD groups, $p<0.05$ or $p<0.01$ (Fig. 2).

Effects of $\beta$-Asarone on Serum Levels of cTNT, IL-1 $\beta$ and Contents of Cardiac Tissue MPO As markers of myocardial damage, cell pyroptosis and local inflammation, the serum levels of cTNT, IL- $1 \beta$ and cardiac tissue contents of MPO were obviously increasing in the I/R group rats. Treating with $\beta$-Asarone could decrease the concentrations of these biomarkers (Table 1).

Effects of $\boldsymbol{\beta}$-Asarone on Cardiac Pathohistology Light microscopy of tissue sections from myocardium of Sham group rats exhibited a normal myofibrillar structure with striations, branched appearance and continuity with adjacent myofibrils. Tissues from the I/R group rats showed obvious myocardial cell swelling, degeneration, loss of transverse striations and infiltration of inflammatory cells. Tissues from rats 


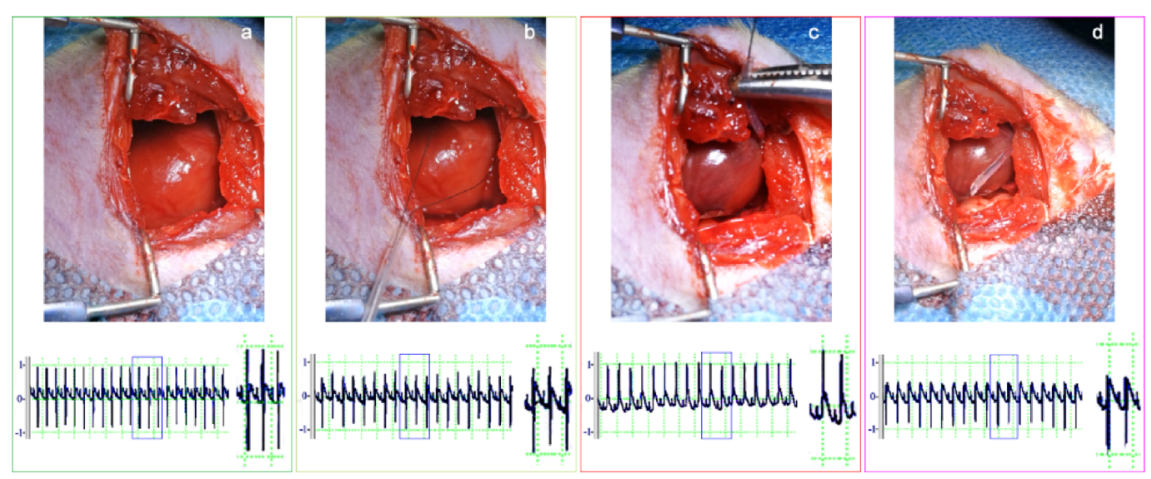

Fig. 1. Rat's Myocardial I/R Model

a: Basic state, b: threading around vessel, c: ligating the vessel, d: loosen the ligation. (Color figure can be accessed in the online version.)
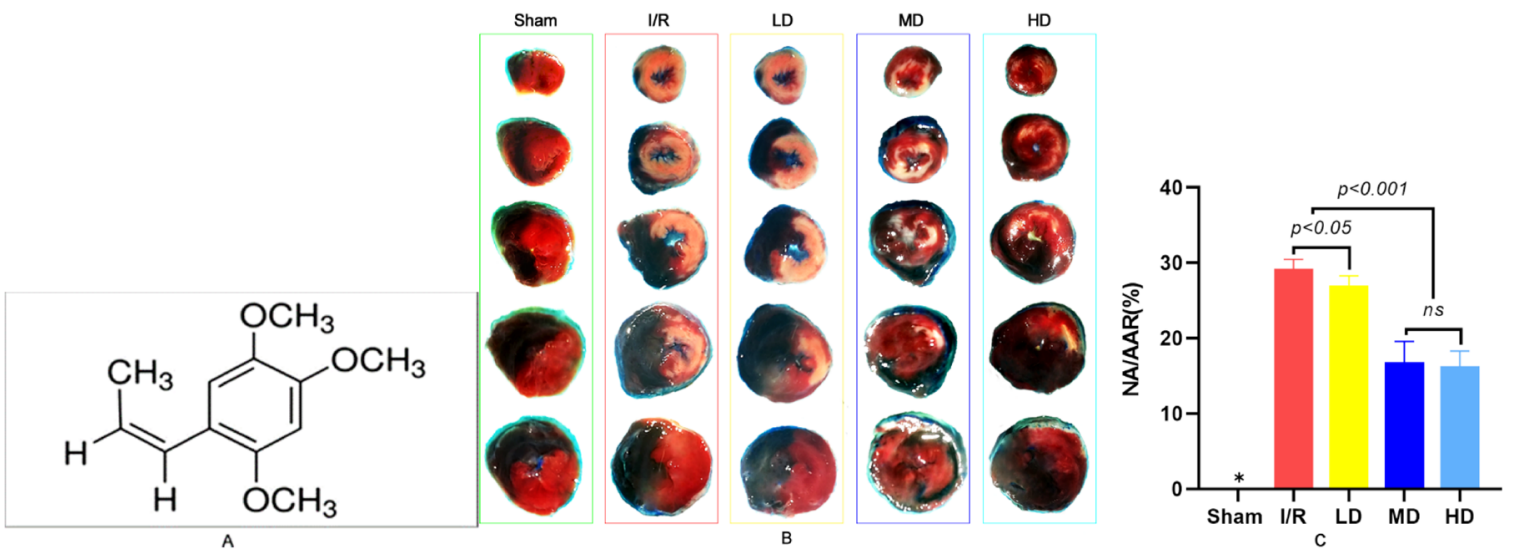

Fig. 2. Effects of $\beta$-Asarone on the Myocardial Infarction Size

A. The chemical structure of $\beta$-Asarone. B. TTC-Evans blue double staining pictures. C. Statistical graph of NA/AAR\% in different groups. *: Sham group nearly had no tissue necrosis. (Color figure can be accessed in the online version.)

Table 1. Biochemical Tests in Different Groups

\begin{tabular}{cccc}
\hline \hline $\begin{array}{c}\text { Tests } \\
\text { Groups }\end{array}$ & cTNT (pg/mL) & IL-1 $\beta$ (ng/L) & MPO (ng/g prot) \\
\hline Sham & $207.8 \pm 96.3$ & $23.9 \pm 3.22$ & $347.6 \pm 36.4$ \\
I/R & $1944.1 \pm 136.1$ & $56.6 \pm 6.01$ & $1352.2 \pm 133.1$ \\
LD & $1772.3 \pm 109.1^{\Delta}$ & $53.1 \pm 4.16$ & $926.3 \pm 86.7^{*}$ \\
MD & $1313.8 \pm 66.8^{*} \triangle$ & $47.6 \pm 3.23^{*} \Delta$ & $763.4 \pm 62.5^{*}$ \\
HD & $1267.3 \pm 85.9^{*}$ & $47.1 \pm 2.83^{*}$ & $720.5 \pm 91.3^{*}$ \\
\hline
\end{tabular}

Sham group had very low while I/R group obviously high values of each tested biochemical markers. Treating with $\beta$-Asarone decreased these values, when compared with I/R group, ${ }^{\wedge}$ meant $p<0.05, * p<0.01 .{ }^{\triangle}$ Values of MD group were much lower than LD group $(p<0.01)$, but had no statistical difference with HD group $(p>0.05)$.

treated with $\beta$-Asarone showed well preserved cardiac muscle structure and less inflammatory cell infiltration (Fig. 3).

Effects of $\boldsymbol{\beta}$-Asarone on NLRP3 Signal Pathway Compared with sham group, the protein levels of NLRP3, ASC and active caspase-1 (P20) were increased in I/R group. After administration of $\beta$-Asarone, the expressions of these proteins or their active fragments were significantly decreased (Fig. 4).

Effects of $\boldsymbol{\beta}$-Asarone on the Expression and Cleavage of GSDMD Compared with the sham group, the protein levels of both full length GSDMD (GSDMD-F) and its cleavage fragments (GSDMD-C) were increased in I/R group. While treating with $\beta$-Asarone, the cleavage level of GSDMD was significantly decreased (Fig. 5).

Effects of $\beta$-Asarone on the Rat Cardiac Function
Compared with the sham group animals, rats in I/R group demonstrated markedly lower LVEF and FS, but higher LVIDs. While administration of $\beta$-Asarone could improve the ultrasonic parameters of left ventricle performance, and preserved the cardiac function as a whole. (Fig. 6, Table 2).

\section{DISCUSSION}

$\beta$-Asarone is the major active ingredient of Acorus tatarinowii Rhizoma, and the latter is a classic traditional Chinese medicine with the main function of "eliminating phlegmatic-statasis," primarily implying anti-inflammation and anti-edema of the affected organ tissue. ${ }^{11)}$ Previous studies demonstrated that the monomeric compound virtually had anti-inflammation and other pharmacological activities. ${ }^{12)}$

In this study, the myocardial I/R model was established and confirmed by ST segment variation of ECG and dynamic changes of myocardial surface color (Supplementary material). Treatment of $\beta$-Asarone alleviated myocardial histological alteration and preserved the cardiac tissue structure, especially alleviating myocardial edema, which also decreased the level of serum cTNT, meaning less cardiac damages. Meanwhile, in vivo TTC-Evans blue double staining really demonstrated the state of tissue necrosis, and the results showed that rats receiving $\beta$-Asarone injection had obviously smaller myocardial infarction areas.

Inflammation was considered as a major driving force in the ischemic process and increasing evidence demonstrated 


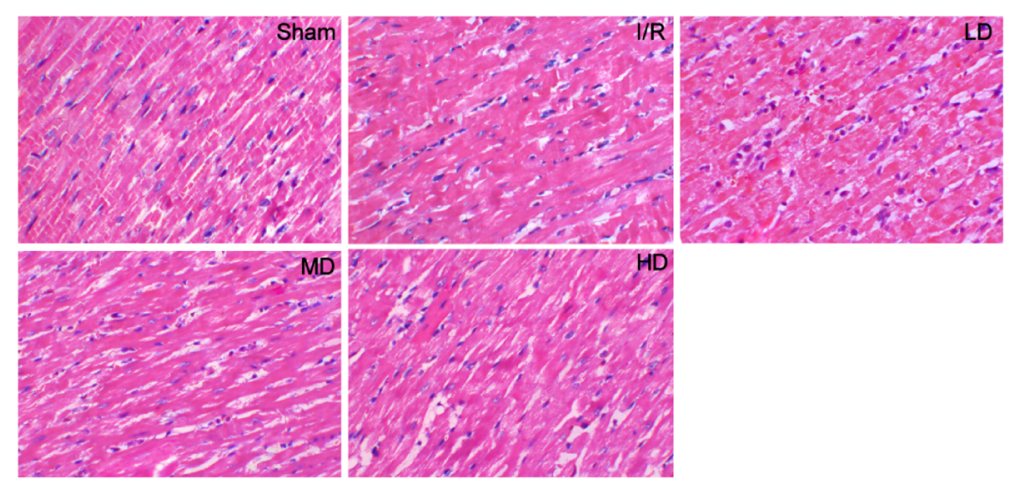

Fig. 3. HE Staining of Left Ventricular Tissue Sections in Different Groups (Color figure can be accessed in the online version.)

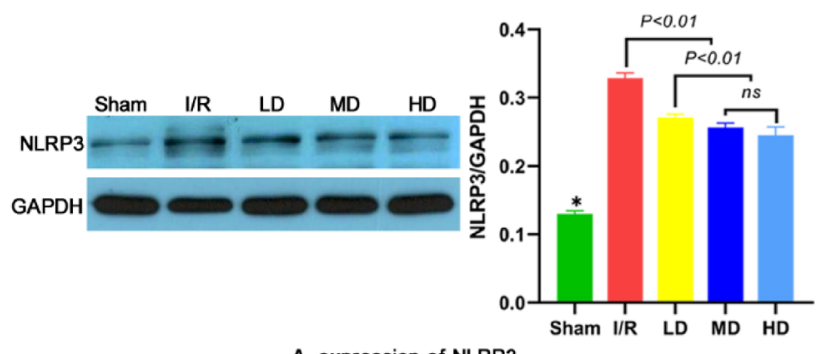

A. expression of NLRP3

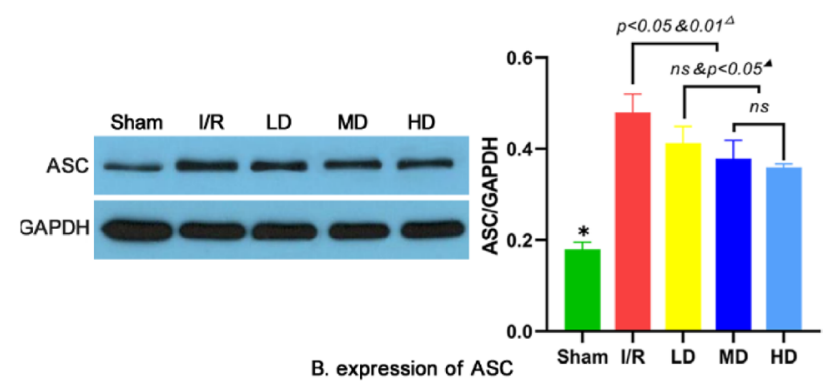

B. expression of ASC

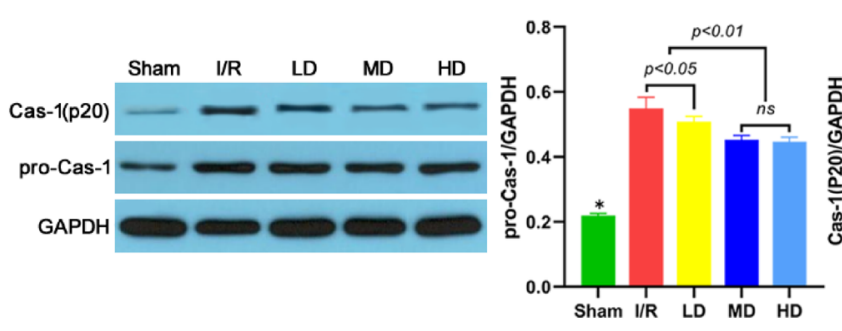

C. expression and activation of caspase-1

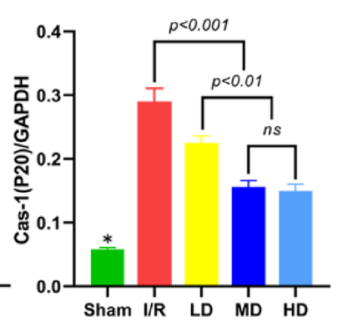

Fig. 4. Expressions of NLRP3, ASC and Activated Caspase-1 (p20)/Pro-caspase-1 in Different Groups

*: compared with other groups, $p<0.001 .{ }^{\Delta}$ : I/R group $v s$. LD group, $p<0.05$; I/R group $v s$. MD or HD group, $p<0.01$. ${ }^{\Delta}$ : value of LD group was no statistical difference compared with MD group, but much higher than HD group, $p<0.05$. (Color figure can be accessed in the online version.)

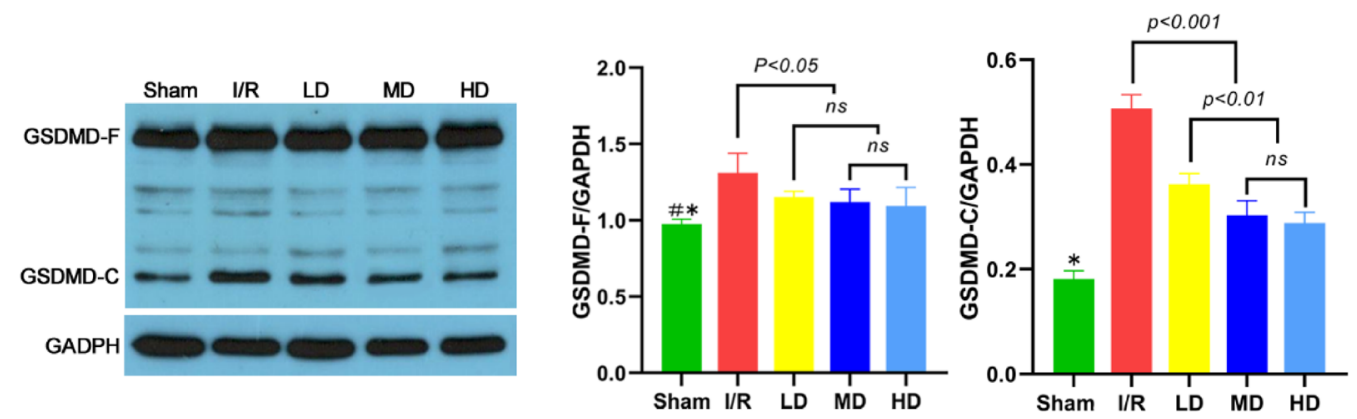

Fig. 5. Expression and Cleavage of GSDMD in Different Groups

\#: sham group $v$ s. HD group, $p<0.05$, and *: compared with other groups, $p<0.01$. (Color figure can be accessed in the online version.)
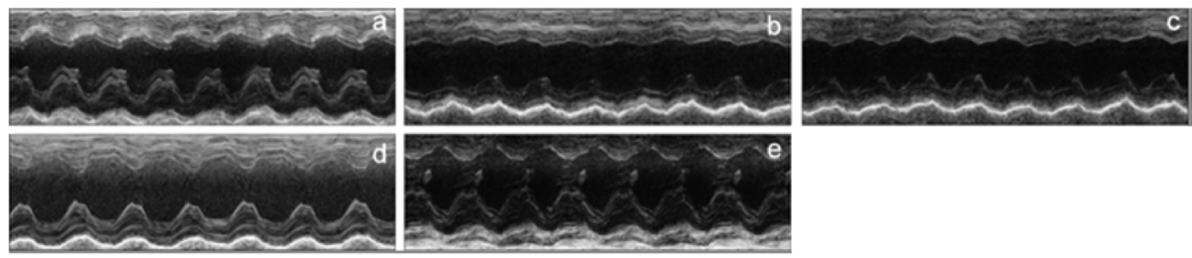

Fig. 6. M-Model Inspection of Echocardiography in Different Groups

a: sham group, b: I/R group, c: LD group, d: MD group, e: HD group. 
Table 2. Parameters of Echocardiography in Different Groups

\begin{tabular}{|c|c|c|c|c|}
\hline $\begin{array}{l}\text { Tests } \\
\text { Groups }\end{array}$ & LVIDd (mm) & LVIDs (mm) & FS $(\%)$ & LVEF (\%) \\
\hline Sham & $5.74 \pm 0.09$ & $2.81 \pm 0.06$ & $53.0 \pm 2.01$ & $77.8 \pm 3.96$ \\
\hline $\mathrm{I} / \mathrm{R}$ & $7.29 \pm 0.22$ & $5.14 \pm 0.19$ & $29.5 \pm 0.96$ & $52.5 \pm 3.83$ \\
\hline LD & $6.55 \pm 0.14^{* \&}$ & $4.13 \pm 0.18^{*}$ & $32.6 \pm 1.87 *$ & $57.7 \pm 3.56^{*}$ \\
\hline MD & $6.32 \pm 0.08^{\mathbf{\Delta}}$ & $3.66 \pm 0.08^{\mathbf{\Delta}}$ & $41.4 \pm 0.95^{\mathbf{\Delta}}$ & $70.4 \pm 2.85^{\mathbf{\Delta}} \Delta$ \\
\hline $\mathrm{HD}$ & $6.23 \pm 0.17^{\mathbf{\Delta} \&}$ & $3.79 \pm 0.09^{\wedge}$ & $42.3 \pm 1.64^{\Lambda}$ & $71.0 \pm 2.99^{\boldsymbol{\Lambda}}$ \\
\hline
\end{tabular}

I/R group had higher LVIDd and LVIDs, but lower FS and LVEF than othe groups. $\beta$-Asarone treatment in all the three groups obviously improved the ultrasonic parameters with lower LVIDd, LVIDs and higher FS, LVEF, when compared with those in the I/R group, * meant $p<0.05,{ }^{\star} p<0.01 .{ }^{\triangle}$ LVIDs, FS and LVEF in MD group exhibited no significant difference with HD group, $p>0.05$. \& : LVIDd in al the three drug administration groups had no statistical difference, $p>0.05$.

that elevated levels of inflammatory biomarkers were related to ischemia and I/R injury. ${ }^{13)} \mathrm{As} \mathrm{I} / \mathrm{R}$ in the heart occured in absence of microorganisms, the inflammatory response was mainly invoked by degenerative or necrotic cells, and amplified by various pro-inflammation cytokines. ${ }^{14,15)}$ This aseprtic inflammatory responses shared similar process to those invoked by invading pathogens. Restoration of blood stream markedly promoted neutrophil sequestration into previously ischemic tissues, then these innate immune cells would produce a nicotinamide adenine dinucleotide phosphate (NADPH) oxidase-dependent respiratory burst and release hydrolytic enzymes via the enzymatic activity of MPO, which induced reperfusion injury that amplified the cellular damage initiated by ischemia. ${ }^{16,17)}$ As the major functional enzyme of neutrophil, MPO also represented existence of the cell itself. ${ }^{18)}$ Through HE staining, our study demonstrated that I/R stimuli resulted in granulocytes infiltration in the cardiac tissue, which were further confirmed as neutrophils by the high level contents of the tissue MPO. While $\beta$-Asarone treatment remarkably reduced the infiltration of these inflammatory cells with decreased tissue MPO contents. So, all discussed above indicated that $\beta$-Asarone had the protection effects probably by inhibition of primary inflammatory response during myocardial I/R stress.

As an important inflammation mediator in the innate immune response, NLRP3 inflammasome played a critical role in myocardial $\mathrm{I} / \mathrm{R}$ injury for it promoted the maturation of inflammatory cytokines like IL- $\beta$ and IL-18, and it had been evidenced that NLRP3 inhibition protected animals against I/R induced cardiac damage. ${ }^{19,20)}$ Typically, NLRP3 pathway was activated through damage associated molecular patterns (DAMPs) by recognition of some endogenous danger signal molecules in a sterile state, or initiated by the combination of soluble ligands and membrane receptors under infectious situation. $^{21)} \mathrm{I} / \mathrm{R}$ in the heart invoked aseprtic inflammatory responses, and study had witnessed that the NLRP3 signal pathway could be activated by endogenous adenosine triphosphate as DAMPs in cardiac fibroblasts. ${ }^{22)}$ Meanwhile, another study observed that cardiac microvascular endothelial cells (CMECs) were also responsible for NLRP3 pathway mediated inflammation via reactive oxygen species and thioredoxininteracting protein (ROS/TXNIP) interaction during $\mathrm{I} / \mathrm{R}$ in heart. ${ }^{23)}$ No matter how it was initiated, a key step for this inflammatory pathway was assembly and activation of the NLRP3 inflammasome complex, in which ASC played a vital role as the adaptor molecule to mediate interaction of NLRP3 protein. And only the assembled NLRP3 inflammasome could activate its functional enzyme - caspase- $1 .{ }^{24)}$ Through analysis of the expression of these relevant proteins, our results showed administration of $\beta$-Asarone could inhibit the NLRP3 signal pathway, decrease recruitment and activation of caspase-1, which suggested its possible function of immunoregulation. Certainly, the exact target of this compound needed further investigation in the future.

Recent studies revealed that the activation of NLRP3 inflammasome not only produced fertile inflammatory cytokines but mediated a kind of necrotic cell death directly, named pyroptosis. ${ }^{25)}$ Pyroptosis was a type of programmed cell death that featured pore formation on the plasma membrane, cell swelling and plasma membrane disruption, so similar to that of necrosis. ${ }^{26}$ This type of cell death was executed by the Gasdermin family proteins, among them GSDMD played an important role, which was classically activated in the NLRP3 signal pathway when cleaved by caspase-1 to release the functional pore-forming N-terminal. ${ }^{27,28)}$ In order to explore whether pyroptosis occurred during $\mathrm{I} / \mathrm{R}$ in heart and the effects of $\beta$-Asarone on it, our study further investigated the expression and activation of this executor. The results demonstrated that I/R stimuli could induce the expression of GSDMD in the rat heart, while $\beta$-Asarone treatment markedly decreased the cleavage and activation of this protein, which was consistent with the level of caspase- 1 activity. And IL- $1 \beta$, a key proinflammatory cytokine which was closely associated with myocardial infarction and myocardial I/R injury, ${ }^{29,30)}$ also acted as an indicator of pyroptosis for its releasing style_— - through the pyroptosis pores in membrane. ${ }^{31)}$ Present results showed that the functional expressions of GSDMD also paralleled with the serum level of IL-1 $\beta$. All these meant I/R in heart induced pyroptosis, but $\beta$-Asarone could decrease the cleavage and activation of GSDMD mostly by inhibition of NLRP3 inflammasome pathway, thus protected the cardiac cell death from that way. Thus, it was rational to speculate that during $\mathrm{I} / \mathrm{R}$ in heart, blood restoration provoking primary inflammatory response caused tissue necrosis, which in turn activated NLRP3 signal pathway and mediated cell's pyroptosis, and released fertile pro-inflammatory cytokines to aggravate and amplify the inflammatory damages. Above results showed that $\beta$-Asarone could inhibit primary inflammatory response and pyroptosis - the two key spots of this vicious circle.

As Heusch and Gersh reviewed recently, mortality from AMI despite widespread access to reperfusion therapy was still substantial for the development of heart failure. ${ }^{32)}$ In order to investigate the effects of $\beta$-Asarone on this adverse outcome, we evaluated left ventricular performance of the animal model by echocardiography measurements at the end of experiments. The results showed that $\beta$-Asarone treatment well preserved the cardiac function with higher LVEF, FS, and lower LVIDd, LVIDs. That indicated this drug probably had the effect to improve prognosis.

\section{CONCLUSION}

Present study demonstrated $\beta$-Asarone alleviated myocardial damage, reduced infarction area and preserved cardiac function after $\mathrm{I} / \mathrm{R}$ in the rat heart. These cardioprotective effects could be attributed to its efffects on suppressing the inflammatory response and decreasing NLRP3 inflamma- 
some induced pryoptosis. Therefore, obtained data suggested that $\beta$-Asarone could be a promising therapeutic or preventive agent for the treatment of $\mathrm{I} / \mathrm{R}$ induced cardiac damage.

Acknowledgments This work was supported by Beijing Natural Science Foundation (7172094), and National Natural Science Foundation (81574036). We also thanked the animal laboratory of Xuanwu Hospital Capital Medical University for supplying experimental apparatus and technical instructions.

Conflict of Interest The authors declare no conflict of interest.

Supplementary Materials The online version of this article contains supplementary materials.

\section{REFERENCES}

1) Benjamin EJ, Virani SS, Callaway CW, et al. Heart disease and stroke statistics-2018 update: a report from the American Heart Association. Circulation, 137, e67-e492 (2018).

2) Hausenloy DJ, Yellon DM. Targeting myocardial reperfusion injury-the search continues. N. Engl. J. Med., 373, 1073-1075 (2015).

3) Hausenloy DJ, Barrabes JA, Botker HE, et al. Ischaemic conditioning and targeting reperfusion injury: a 30 year voyage of discovery. Basic Res. Cardiol., 111, 70 (2016).

4) Kalogeris T, Baines CP, Krenz M, Korthuis RJ. Ischemia-reperfusion. Compr. Physiol., 7, 113-170 (2016).

5) Oerlemans MI, Koudstaal S, Chamuleau SA, de Kleijn DP, Doevendans PA, Sluijter JP. Targeting cell death in the reperfused heart: pharmacological approaches for cardioprotection. Int. J. Cardiol., 165, 410-422 (2013)

6) Liu W, Zhang B, Xin Z, Ren D, Yi L. GC-MS Fingerprinting combined with chemometric methods reveals key bioactive components in Acori tatarinowii Rhizoma. Int. J. Mol. Sci., 18, 18 (2017).

7) Xiao X, Xu X, Li F, Xie G, Zhang T. Anti-inflammatory treatment with beta-asarone improves impairments in social interaction and cognition in MK-801 treated mice. Brain Res. Bull., 150, 150-159 (2019).

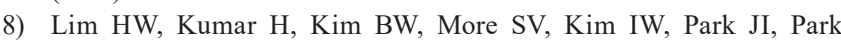
SY, Kim SK, Choi DK. beta-Asarone (cis-2,4,5-trimethoxy-1-allyl phenyl), attenuates pro-inflammatory mediators by inhibiting NFkappaB signaling and the JNK pathway in LPS activated BV-2 microglia cells. Food Chem. Toxicol., 72, 265-272 (2014).

9) Lindsey ML, Bolli R, Canty JJ, Du XJ, Frangogiannis NG, Frantz S, Gourdie RG, Holmes JW, Jones SP, Kloner RA, Lefer DJ, Liao R, Murphy E, Ping P, Przyklenk K, Recchia FA, Schwartz LL, Ripplinger CM, Van Eyk JE, Heusch G. Guidelines for experimental models of myocardial ischemia and infarction. Am. J. Physiol. Heart Circ. Physiol., 314, H812-H838 (2018).

10) Li LH, Chen WH, Zheng H. An optimized modification of TTC staining method for better detection of myocardial ischemia-reperfusion injury in rats. Acta Lab. Anim. Sci. Sin., 24, 75-78 (2014).

11) Li J, Li X, Gao YY, Dong WR, Pang YH, Cao MM. Research progress of Chinese herbal Acorus tatarinowii Schott. JOURNAL OF LIAONING UNIVERSITY OF TCM, 21, 13-17 (2019).

12) Chellian R, Pandy V, Mohamed Z. Pharmacology and toxicology of alpha- and beta-asarone: a review of preclinical evidence. Phytomedicine, 32, 41-58 (2017).

13) Saxena A, Russo I, Frangogiannis NG. Inflammation as a therapeutic target in myocardial infarction: learning from past failures to meet future challenges. Transl. Res., 167, 152-166 (2016).

14) Frangogiannis NG. Inflammation in cardiac injury, repair and regeneration. Curr. Opin. Cardiol., 30, 240-245 (2015).

15) Ioannou A, Dalle LJ, Tsokos GC. Immunopathogenesis of ischemia/ reperfusion-associated tissue damage. Clin. Immunol., 141, 3-14 (2011).

16) $\overline{\text { Boros }} \mathrm{P}$, Bromberg JS. New cellular and molecular immune pathways in ischemia/reperfusion injury. Am. J. Transplant., 6, 652-658 (2006).

17) Ford DA. Lipid oxidation by hypochlorous acid: chlorinated lipids in atherosclerosis and myocardial ischemia. Clin. Lipidol., 5, 835-852 (2010).

18) Marcinkiewicz J, Walczewska M. Neutrophils as sentinel cells of the immune system: a role of the MPO-halide-system in innate and adaptive immunity. Curr. Med. Chem., 26 (2019).

19) Marchetti C, Chojnacki J, Toldo S, Mezzaroma E, Tranchida N, Rose SW, Federici M, Van Tassell BW, Zhang S, Abbate A. A novel pharmacologic inhibitor of the NLRP3 inflammasome limits myocardial injury after ischemia-reperfusion in the mouse. J. Cardiovasc. Pharmacol., 63, 316-322 (2014).

20) Toldo S, Mauro AG, Cutter Z, Van Tassell BW, Mezzaroma E, Del BM, Prestamburgo A, Potere N, Abbate A. The NLRP3 inflammasome inhibitor, OLT1177 (Dapansutrile), reduces infarct size and preserves contractile function after ischemia-reperfusion injury in the mouse. J. Cardiovasc. Pharmacol., 73, 215-222 (2019).

21) Gross O, Thomas CJ, Guarda G, Tschopp J. The inflammasome: an integrated view. Immunol. Rev., 243, 136-151 (2011).

22) Sandanger $O$, Ranheim $T$, Vinge LE, Bliksoen $M$, Alfsnes $K$, Finsen AV, Dahl CP, Askevold ET, Florholmen G, Christensen G, Fitzgerald KA, Lien E, Valen G, Espevik T, Aukrust P, Yndestad A. The NLRP3 inflammasome is up-regulated in cardiac fibroblasts and mediates myocardial ischaemia-reperfusion injury. Cardiovasc. Res., 99, 164-174 (2013).

23) Liu Y, Lian K, Zhang L, Wang R, Yi F, Gao C, Xin C, Zhu D, Li Y, Yan W, Xiong L, Gao E, Wang H, Tao L. TXNIP mediates NLRP3 inflammasome activation in cardiac microvascular endothelial cells as a novel mechanism in myocardial ischemia/reperfusion injury. Basic Res. Cardiol., 109, 415 (2014).

24) Guo H, Callaway JB, Ting JP. Inflammasomes: mechanism of action, role in disease, and therapeutics. Nat. Med., 21, 677-687 (2015).

25) Kesavardhana S, Kanneganti TD. Mechanisms governing inflammasome activation, assembly and pyroptosis induction. Int. Immunol., 29, 201-210 (2017).

26) Fink SL, Cookson BT. Apoptosis, pyroptosis, and necrosis: mechanistic description of dead and dying eukaryotic cells. Infect. Immun., 73, 1907-1916 (2005).

27) Lamkanfi M, Dixit VM. Inflammasomes and their roles in health and disease. Annu. Rev. Cell Dev. Biol., 28, 137-161 (2012).

28) Liu X, Zhang Z, Ruan J, Pan Y, Magupalli VG, Wu H, Lieberman J. Inflammasome-activated gasdermin D causes pyroptosis by forming membrane pores. Nature, 535, 153-158 (2016).

29) Dinarello CA. A clinical perspective of IL-lbeta as the gatekeeper of inflammation. Eur. J. Immunol., 41, 1203-1217 (2011).

30) Buckley LF, Abbate A. Interleukin-1 blockade in cardiovascular diseases: from bench to bedside. BioDrugs, 32, 111-118 (2018).

31) He WT, Wan H, Hu L, Chen P, Wang X, Huang Z, Yang ZH, Zhong CQ, Han J. Gasdermin D is an executor of pyroptosis and required for interleukin-1beta secretion. Cell Res., 25, 1285-1298 (2015).

32) Heusch G, Gersh BJ. The pathophysiology of acute myocardial infarction and strategies of protection beyond reperfusion: a continual challenge. Eur. Heart J., 38, 774-784 (2017). 